10) 1991 Kluwer Academic Publishers. Printed in the Netherlands.

\title{
A New Quantum Group Associated with a 'Nonstandard' Braid Group Representation
}

\author{
NAIHUAN JING * \\ School of Mathematics, Institute for Advanced Study, Princeton, NJ 08540, U.S.A. \\ MO-LIN GE ${ }^{\star \star}$ \\ Institute for Theoretical Physics, State Universtty of New York at Stony Brook, Stony Brook, \\ NY 11794-3840, U.S.A. \\ and \\ YONG-SHI WU \\ School of Natural Sciences, Institute for Adianced Study, Princeton, NJ 08540, U.S.A.
}

(Received: 23 April 1990; revised version: 20 September 1990)

\begin{abstract}
A new quantum group is derived from a 'nonstandard' braid group representation by employing the Faddeev-Reshetıkhin-Takhtajan constructive method. The classical limit is not a Lie superalgebra, despite relations like $x^{2}=y^{2}=0$. We classify all finite-dimensional irreducible representations of the new Hopf algebra and find only one- and two-dimensional ones.
\end{abstract}

AMS subject classifications (1980). 16A24, 17B55, 20GXX, 22E65.

\section{Introduction}

Recently, the so-called quantum groups [1,2] have attracted a lot of attention. Originally they were invented for a systematic procedure for solving the quantum Yang-Baxter equations [3]. During the last two years, hidden quantum group structures have been revealed in various physically interesting models and theories, such as conformal field theory [4], topological Chern-Simons gauge theory [5], and integrable statistical lattice models [6].

Mathematically, quantum groups have been characterized by Drinfeld [1] as noncommutative and noncocommutative Hopf algebras. However, almost all known concrete examples have been restricted to the 'standard' $q$-deformation of usual symmetrizable Kac-Moody Lie algebras or groups, except the generalizations to Lie superalgebras or groups. In this Letter, we present the explicit construction of some new quantum groups (or noncommutative and noncocommutative Hopf algebras), which are associated with certain 'nonstandard' braid group representa-

* Present address: Department of Mathematıcs, University of Michıgan, Ann Arbor, MI 48109, U.S.A.

$\star \star$ Permanent address: Theoretical Physics Division, Nankai Institute of Mathematics, Tianjin 300071,

P.R. China.

‡ On leave from Department of Physics, University of Utah, Salt Lake City, UT 84112 , U S.A. 
tions (BGR's). By now, it has become well-known that many BGRs can be obtained from the universal $R$-matrix $[7,8]$ for the standard $q$-deformation of usual Lie algebras; we refer to such BGRs as 'standard' ones. A lot of 'nonstandard' BGRs, which cannot be obtained in this way, have been available by the direct method in our previous works $[9,10]$. There we have asked: What are the quantum groups associated with these new 'nonstandard' BGRs? This Letter represents the first step in this direction.

As pointed out by Faddeev, Reshetikhin and Takhtajan [12], the usual quantum groups (or the standard $q$-deformation of usual Lie algebras) can be obtained by a definite, purely algebraic procedure from appropriate standard BGRs or $R$ matrices, viewing the latter as a sort of structure constants and the braid YangBaxter relations they satisfy as the generalized Jacobi identities. In this Letter, we will apply their procedure to the simplest nonstandard or BGR $[9,10]$

$$
\check{R}^{+}=\left(\begin{array}{cccc}
q & 0 & 0 & 0 \\
0 & 0 & 1 & 0 \\
0 & 1 & q-q^{-1} & 0 \\
0 & 0 & 0 & -q^{-1}
\end{array}\right) .
$$

We determine both the Hopf algebra and its dual associated with this BGR. (Some previous discussions can be found in $[13,14]$.) These Hopf algebras are certainly beyond the usual ones associated with Lie algebras or superalgebras. Particularly, their representation theory does not bear any resemblance to that of usual quantum groups, in that the only finite-dimensional irreducible representations are one- and two-dimensional ones. A complete classification of two-dimensional irreducible representations and their fusion rules will be given below. An interesting question is what would be the two-dimensional conformal field theory with this quantum group as hidden symmetry (or chiral exchange algebra).

Another motivation comes from a close relation of the $R$-matrix of (1.1) with the Alexander-Conway polynomial in the knot theory $[13,14]$. We expect our new quantum group will shed more light on the complete relationship with the Alexander-Conway polynomial.

\section{Deriving New Quantum Group $X_{q}(2)$}

Following [12], we consider the equations

$$
\begin{aligned}
& \left(L^{( \pm)} \otimes L^{( \pm)}\right) \check{R}^{+}=\check{R}^{+}\left(L^{( \pm)} \otimes L^{( \pm)}\right), \\
& \left(L^{(-)} \otimes L^{(+)}\right) \check{R}^{+}=\check{R}^{+}\left(L^{(+)} \otimes L^{(-)}\right),
\end{aligned}
$$

with $\check{R}_{a b, c d}$ given by Equation (1.1). Here, we have adopted the following conventions $(a, b, c, d=1,2)$ :

$$
\begin{array}{ll}
(A \otimes B)_{a b, c d}=A_{a c} B_{b d}, & \\
\check{R}_{11.11}^{+}=q, \quad \check{R}_{22,22}^{+}=-q^{-1}, \quad \check{R}_{12,21}^{+}=\check{R}_{21,12}^{+}=1, \\
\check{R}_{21,21}^{+}=q-q^{-1}, \quad \check{R}_{a b, c d}^{+}=0 & \text { otherwise. }
\end{array}
$$


As in [12], $L^{(+)}$and $L^{(-)}$are respectively taken to be upper- and lower-triangular $2 \times 2$ matrices with operator entries

$$
L_{21}^{(+)}=0, \quad L_{12}^{(-)}=0 .
$$

It is easy to check that this is compatible with Equations (2.1) and (2.2). By straightforward calculation, it is easily seen that Equations (2.1) and (2.2) lead to the following relations.

$$
\begin{aligned}
& {\left[L_{a d}^{( \pm)}, L_{h b}^{( \pm)}\right]=0,} \\
& L_{11}^{(+)} L_{12}^{(+)}=q^{-1} L_{12}^{(+)} L_{11}^{(+)}, \quad L_{22}^{(+)} L_{12}^{(+)}=-q^{-1} L_{12}^{(+)} L_{22}^{(+)}, \\
& L_{11}^{(-)} L_{21}^{(-)}=q^{-1} L_{21}^{(-)} L_{11}^{(-)}, \quad L_{22}^{(-)} L_{21}^{(-)}=-q^{-1} L_{21}^{(-)} L_{22}^{(-)}, \\
& \left(L_{12}^{(+)}\right)^{2}=0, \quad\left(L_{21}^{(-)}\right)^{2}=0, \\
& L_{11}^{(+)} L_{21}^{(-)}=q L_{21}^{(-)} L_{11}^{(+)}, \quad L_{22}^{(+)} L_{21}^{(-)}=-q L_{21}^{(-)} L_{22}^{(+)}, \\
& L_{11}^{(-)} L_{12}^{(+)}=q L_{12}^{(+)} L_{11}^{(-)}, \quad L_{22}^{(-)} L_{12}^{(+)}=-q L_{12}^{(+)} L_{22}^{(-)}, \\
& {\left[L_{12}^{(+)}, L_{21}^{(-)}\right]=\left(q-q^{-1}\right)\left(L_{22}^{(-)} L_{11}^{(+)}-L_{22}^{(+)} L_{11}^{(-)}\right) .}
\end{aligned}
$$

It is easy to verify that

$$
\begin{aligned}
& \left(L_{22}^{(-)} L_{22}^{(+)}\right) L_{a b}^{( \pm)}=L_{a b}^{(+)}\left(L_{22}^{(-)} L_{22}^{(+)}\right), \\
& \left(L_{11}^{(-)} L_{11}^{(+)}\right) L_{a b}^{( \pm)}=L_{a b}^{( \pm)}\left(L_{11}^{(-)} L_{11}^{(+)}\right),
\end{aligned}
$$

i.e. $L_{a a}^{( \pm)} L_{a a}^{(\mp)}=L_{a a}^{(\mp)} L_{a a}^{( \pm)}$(no sum) commute with $L^{( \pm)}$. So we can choose

$$
L_{11}^{(-)} L_{11}^{(+)}=L_{11}^{(+)} L_{11}^{(-)}=L_{22}^{(-)} L_{22}^{(+)}=L_{22}^{(+)} L_{22}^{(-)}=1 \text {. }
$$

Writing

$$
L^{(+)}=\left(\begin{array}{cc}
k^{-1} & -\left(q-q^{-1}\right) X \\
0 & \xi
\end{array}\right), \quad L^{(-)}=\left(\begin{array}{cc}
k & 0 \\
\left(q-q^{-1}\right) Y & \xi^{-1}
\end{array}\right),
$$

then we have the following relations

$$
\begin{aligned}
& X^{2}=Y^{2}=0, \\
& {[k, \xi]=0,} \\
& k X k^{-1}=q X, \quad \xi X \xi^{-1}=-q^{-1} X, \\
& k Y k^{-1}=q^{-1} Y, \quad \xi Y \xi^{-1}=-q Y, \\
& {[X, Y]=\left(k \xi-k^{-1} \xi^{-1}\right) /\left(q-q^{-1}\right) .}
\end{aligned}
$$

Denote by $X_{q}(2)$ the algebras generated by the elements $1, k^{ \pm 1}, \xi^{ \pm 1}, X$ and $Y$. One can introduce a coproduct in $X_{q}(2)$ by

$$
\begin{aligned}
& \Delta\left(k^{ \pm 1}\right)=k^{ \pm 1} \otimes k^{ \pm 1}, \quad \Delta\left(\xi^{ \pm 1}\right)=\xi^{ \pm 1} \otimes \xi^{ \pm 1} . \\
& \Delta(X)=X \otimes k+\xi^{-1} \otimes X \\
& \Delta(Y)=Y \otimes \xi+k^{-1} \otimes Y .
\end{aligned}
$$


One thus easily verifies, for example,

$$
[\Delta(X), \Delta(Y)]=\Delta([X, Y]), \quad(\Delta(X))^{2}=\Delta\left(X^{2}\right)=0 .
$$

Furthermore, one can define a counit $\varepsilon$ and antipode $S$ as follows

$$
\begin{aligned}
& \varepsilon(X)=\varepsilon(Y)=0, \quad \varepsilon(k)=\varepsilon(\xi)=1, \\
& S(k)=k^{-1}, \quad S(\xi)=\xi^{-1}, \\
& S(X)=-\xi X k^{-1}, \quad S(Y)=-k Y \xi^{-1} .
\end{aligned}
$$

$\varepsilon$ is an algebra homomorphism and $S$ an anti-homomorphism. Therefore, the algebra $X_{q}(2)$ becomes a Hopf algebra.

The question of what is the quantum group (or Hopf algebra) associated with the $\check{R}$-matrix (1.1) has also been studied in [13]; there, a different Hopf algebra is proposed. Here, our Hopf algebra is derived from the more systematic and constructive method given in [12]. In particular, we have relations $X^{2}=Y^{2}=0$, which does not appear in the Hopf algebra proposed in [13]. As we will see below, these relations are necessary for the dual of our $X_{4}(2)$ algebra to have a coproduct. Also, we note that if we remove the relation $X^{2}=Y^{2}=0$, we still get a Hopf algebra. Denote it by $\hat{X}_{q}(2)$. A theorem, which is going to be proved in the Appendix, tells us that in any nontrivial irreducible representation of $\hat{X}_{q}(2)$ that has both highest and lowest weight vectors, one must have $X^{2}=Y^{2}=0$.

Although $X^{2}=Y^{2}=0$ looks like fermionic (or Grasssmannian) relations, the classical limit of $X_{q}(2)$ is not a Lie superalgebra. For we have a commutation (rather than anti-commutation) relation $(2.19-2.20)$ for $X$ and $Y$ with $k, \xi$.

\section{Dual of $X_{q}(2)$}

Now let us determine the dual of the Hopf algebra $X_{q}(2)$ obtained in the last section. A rigorous way is to view it as a quantized formal series Hopf algebra [1], realized as the dual of $X_{4}(2)$.

Consider the fundamental representation $V_{0}$ of $X_{q}(2)$ on $\mathbb{C}^{2}$ given by

$$
\begin{aligned}
& X=\left(\begin{array}{cc}
0 & -1 \\
0 & 0
\end{array}\right), \quad Y=\left(\begin{array}{ll}
0 & 0 \\
1 & 0
\end{array}\right), \\
& k=\left(\begin{array}{cc}
q^{1 / 2} & 0 \\
0 & q^{-1 / 2}
\end{array}\right), \quad \xi=\left(\begin{array}{cc}
q^{-3 / 2} & 0 \\
0 & -q^{-1 / 2}
\end{array}\right) .
\end{aligned}
$$

The matrix elements $x_{i j}$ of $V_{0}$, i.e. $a\left(\in X_{q}(2)\right) \mapsto\left(x_{i}(a)\right) \in$ End $\left(\mathbb{C}^{2}\right)$, are complex linear functionals on $X_{4}(2)$. The natural product on $X_{4}(2)^{*}$ is defined via

$$
f \cdot g(a)=(f \otimes g) \circ(\Delta(a)),
$$

where $f, g \in X_{q}(2), a \in X_{q}(2)$. 
It is an easy exercise to verify the following relations

$$
\begin{aligned}
& x_{11} x_{12}=q x_{12} x_{11}, \quad x_{11} x_{21}=q x_{21} x_{11}, \\
& x_{22} x_{12}=-q x_{12} x_{22}, \quad x_{22} x_{21}=-q x_{21} x_{22}, \\
& x_{12} x_{21}=x_{21} x_{12}, \\
& x_{11} x_{22}-x_{22} x_{11}=\left(q-q^{-1}\right) x_{12} x_{21} .
\end{aligned}
$$

One can add 1 into $X_{q}(2)^{*}$ by

$$
1(a)=\varepsilon(a), \quad a \in X_{4}(2) .
$$

So far, this is good, even for the algebra $\hat{X}_{4}(2)$. However, we are only interested in the dual Hopf algebra of $X_{q}(2)$. Denote by $\tilde{X}_{4}(2)$ the algebra generated by the elements $1, x_{i j}, x_{11}^{-1}, x_{22}^{-1}$ subject to the relations in Equations (3.3)-(3.6) plus $x_{u} x_{t r}^{-1}=x_{n}^{-1} x_{u}=1$. We equip it with a Hopf algebra structure by the induced coproduct $\Delta$ :

$$
\Delta\left(x_{l j}\right)=\sum_{l=1}^{2} x_{l l} \otimes x_{l l},
$$

which has the following property

$$
(a b)(x)=(a \otimes b)(\Delta(x))
$$

Having done this, we find out that we have to add more relations to the algebra structure of $\tilde{X}_{q}(2)$. To see this point, we calculate that, for example,

$$
\Delta\left(x_{12}, x_{22}\right)+q^{-1} \Delta\left(x_{22}, x_{12}\right)=\left(1+q^{-2}\right) x_{11} x_{12} \otimes\left(x_{12}\right)^{2} .
$$

Thus, to make the Hopf algebra structure $\tilde{X}_{q}(2)$ work, we are forced to add the following relations

$$
\left(x_{12}\right)^{2}=\left(x_{21}\right)^{2}=0 .
$$

The relation (3.11) is implicitly connected to the similar relations $X_{q}(2)$. As $X^{2}=Y^{2}=0$ are bounded by the other relations of the algebra in representations, the relation (3.11) are restricted by the coproduct and the various relations in the algebra structure of $\tilde{X}_{q}(2)$. The later relations are actually determined by the same relations in the algebra $X_{q}(2)$ that govern the relations $X^{2}=Y^{2}=0$ (cf. Appendix).

The algebra $\tilde{X}_{q}(2)$ has the antipode $\gamma$ induced from that of $X_{q}(2)$ in the following manner. For $a \in X_{q}(2)$ we define $\gamma$ by

$$
\gamma\left(x_{t j}\right)(a)=x_{i j}(S(a))
$$

and extend

$$
\gamma \quad \text { to } \tilde{X}_{4}(2) \text { by } \gamma(a b)=\gamma(b) \gamma(a) .
$$


It is easy to see that ${ }^{\star}$,

$$
\gamma\left(\begin{array}{ll}
x_{11} & x_{12} \\
x_{21} & x_{22}
\end{array}\right)=\left(\begin{array}{cc}
x_{11}^{-1}\left(1+q x_{12}\left(x_{11} x_{22}\right)^{-1} x_{21}\right) & q\left(x_{22} x_{11}\right)^{-1} x_{12} \\
-q\left(x_{11} x_{22}\right)^{-1} x_{21} & x_{22}^{-1}\left(1-q x_{21}\left(x_{22} x_{11}\right)^{-1} x_{12}\right)
\end{array}\right) .
$$

Another way of obtaining $\tilde{X}_{q}(2)$ is to solve the following equations, $a$ la [12],

$$
\breve{R}(x \otimes x)=(x \otimes x) \breve{R},
$$

where $x=\left(x_{i}\right)_{1 \leqslant i, j \leqslant 2}$ and $\check{R}=P R=P \check{R}^{+} P$. Here, $P$ is the transposition in $\operatorname{End}\left(\mathbb{C}^{2} \otimes \mathbb{C}^{2}\right)$, i.e. $P(u \otimes v)=v \otimes u$.

It is straightforward to check that Equation (3.15) gives the same relations as Equations (3.3)-(3.6) and (3.11).

We remark that the same algebra $\tilde{X}_{q}(2)$ without antipode is obtained in [14] from a different context.

\section{Representations of $X_{q}(2)$}

Now we proceed to study the representation theory of the Hopf algebra $X_{4}(2)$ we defined in Section 2. The fundamental representation $V_{0}$ of $X_{4}(2)$ is given by Equation (3.1), and the corresponding $R$-matrix is

$$
R=\left[\begin{array}{cccc}
q & 0 & 0 & 0 \\
0 & 1 & 0 & 0 \\
0 & q-q^{-1} & 1 & 0 \\
0 & 0 & 0 & -q^{-1}
\end{array}\right]=\check{R}^{+} P
$$

Let us consider the tensor product $V_{0} \otimes V_{0}$, which is a four-dimensional representation of $X_{y}(2)$ via the coproduct

$$
\forall a \in X_{4}(2), \quad \pi(a)=\left(\pi_{0} \otimes \pi_{0}\right) \Delta(a)
$$

For example, in $V_{0} \otimes V_{0}$, the generator $X$ is represented by

$$
\begin{aligned}
\pi(X) & =\pi_{0}(X) \otimes \pi_{0}(k)+\pi_{0}\left(\xi^{-1}\right) \otimes \pi_{0}(X) \\
& =\left(\begin{array}{rr}
0 & -1 \\
0 & 0
\end{array}\right) \otimes\left(\begin{array}{cc}
q^{1 / 2} & 0 \\
0 & q^{-1 / 2}
\end{array}\right)+\left(\begin{array}{cc}
q^{3 / 2} & 0 \\
0 & -q^{1 / 2}
\end{array}\right) \otimes\left(\begin{array}{cr}
0 & -1 \\
0 & 0
\end{array}\right) .
\end{aligned}
$$

It is easy to see that this four-dimensional representation is reducible and can be decomposed into two inequivalent two-dimensional irreducible representations:

$$
V_{0} \otimes V_{0}=V_{1} \oplus V_{2}
$$

\footnotetext{
* We are grateful to Prof. T. A. Springer for helping us on this formula.
} 
Suppose $\left\{e_{1}, e_{2}\right\}$ is the basis of $V_{0}$ as $\mathbb{C}^{2}$, then the bases in $V_{1}$ and $V_{2}$ are, respectively, given by

$$
\begin{array}{ll}
V_{1}: & e_{1} \otimes e_{1}, e_{1} \otimes e_{2}+q^{-1} e_{2} \otimes e_{1}, \\
V_{2}: & e_{1} \otimes e_{2}-q e_{2} \otimes e_{1}, e_{2} \otimes e_{2} .
\end{array}
$$

The matrix representations of the generators are given by

$$
\begin{aligned}
V_{1}: & \pi_{1}(X)=\left(\begin{array}{cc}
0 & -\left(q^{-1,2}+q^{3 / 2}\right) \\
0 & 0
\end{array}\right), \quad \pi_{2}(Y)=\left(\begin{array}{cc}
0 & 0 \\
q^{-1 / 2} & 0
\end{array}\right), \\
\pi_{1}(k) & =\left(\begin{array}{ll}
q & 0 \\
0 & 1
\end{array}\right), \quad \pi_{1}(\xi)=\left(\begin{array}{cc}
q^{-3} & 0 \\
0 & -q^{-2}
\end{array}\right), \\
V_{2}: \pi_{2}(X) & =\left(\begin{array}{cc}
0 & -q^{-1 / 2} \\
0 & 0
\end{array}\right), \quad \pi_{2}(Y)=\left(\begin{array}{cc}
0 & 0 \\
-\left(q^{-1 / 2}+q^{3 / 2}\right) & 0
\end{array}\right), \\
\pi_{2}(k) & =\left(\begin{array}{cc}
1 & 0 \\
0 & q^{-1}
\end{array}\right), \quad \pi_{2}(\xi)=\left(\begin{array}{cc}
-q^{-2} & 0 \\
0 & q^{-1}
\end{array}\right) .
\end{aligned}
$$

The three two-dimensional representations $V_{0}, V_{1}, V_{2}$ are inequivalent, since the $\operatorname{tr} k$ (or $\operatorname{tr} \xi$ ) in them are not the same. If we keep going on by taking the tensor product with more $V_{0}^{\prime} s$, we will get infinitely many two-dimensional irreducible representations.

In general, we have the following theorems in the representation theory of $X_{q}(2)$ :

THEOREM 1. An irreducible finite-dimensional representation $V$ of the Hopf algebra $X_{q}(2)$ is either one-or two-dimensional. Only two-dimensional representations are nontrivial.

Proof. Since $X^{2}=0$, null $(X)=\{v \in V \mid X v=0\} \neq \emptyset$. Note that null $(X)$ is invariant under the action of both $k$ and $\xi$. Similar statements are true for null $(Y)$.

Case 1: $\operatorname{null}(X) \cap \operatorname{null}(Y) \neq \emptyset$.

Pick a vector $v_{1} \in$ null $(X) \cap$ null $(Y)$ which is a simultaneous eigenvector of $k$ and $\xi: k v_{1}=k_{1} v_{1}, \xi v_{1}=\xi_{1} v_{1}$. Since $\left\{v_{1}\right\}$ forms a one-dimensional invariant subspace of $V$, by the irreducibility of $V$ we see that $V=\left\{v_{1}\right\}$. Namely $V$ is one-dimensional. $[X, Y] v=0$ implies that $\left(k_{1} \xi_{1}\right)^{2}=1$.

Case 2: $\operatorname{null}(X) \cap \operatorname{null}(Y)=\emptyset$.

Pick a vector $v_{1} \in \operatorname{null}(X)$ which is a simultaneous eigenvector of $k$ and $\xi: k v_{1}=k_{1} v_{1}, \xi v_{1}=\xi_{1} v_{1}$. Consider $u_{1} \equiv Y v_{1}$, then

$$
X u_{1}=[X, Y] v_{1}=\left(k_{1} \xi_{1}-k_{1}^{-1} \xi_{1}^{-1}\right) v_{1} /\left(q-q^{-1}\right) \neq 0,
$$

otherwise $u_{1} \in \operatorname{null}(X) \cap \operatorname{null}(Y)$. So $k_{1} \xi_{1} \neq\left(k_{1} \xi_{1}\right)^{-1}$. Thus, the subspace spanned by $v_{1}$ and $u_{1} \equiv Y v_{1}$ is invariant under the algebra $X_{q}(2)$. The irreducibility of $V$ tells us that $V$ is just this two-dimensional space spanned by $v_{1}$ and $Y v_{1}$. 
From the proof of this theorem, we see that all one-dimensional representations have the form

$$
X=(0), \quad Y=(0), \quad k=\left(k_{1}\right), \quad \xi=\left(\xi_{1}\right)\left(k_{1}^{2} \xi_{1}^{2}=1\right) .
$$

Any two-dimensional representation $V_{l}$ is equivalent to the following form

$$
\begin{aligned}
& X=\left(\begin{array}{cc}
0 & x_{1} \\
0 & 0
\end{array}\right), \quad Y=\left(\begin{array}{ll}
0 & 0 \\
y_{\imath} & 0
\end{array}\right), \\
& k=\left(\begin{array}{cc}
k_{\imath} & 0 \\
0 & q^{-1} k_{l}
\end{array}\right), \quad \xi=\left(\begin{array}{cc}
\xi_{l} & 0 \\
0 & -q \xi_{l}
\end{array}\right)
\end{aligned}
$$

with

$$
x_{l} y_{i}\left(q-q^{-1}\right)=k_{l} \xi_{t}-k_{l}^{-1} \xi_{l}^{-1} \neq 0
$$

where $x_{t}, y_{l}, k_{t}, \xi_{l} \in \mathbb{C}^{\times}$. Variance of $x_{\imath}$ and $y_{t}$ subject to Equation (4.6) does not change the equivalence of $V_{l}$. In particular, we can choose

$$
y_{i}=1, \quad x_{i}=\frac{k_{i} \xi_{1}-k_{i}^{-1} \xi_{t}^{-1}}{q-q^{-1}}
$$

THEOREM 2. Two representations $V_{1}$ and $V_{2}$ are equivalent to each other iff $\operatorname{tr}_{V_{1}}(k)=\operatorname{tr}_{v_{2}}(k)$ and $\operatorname{tr}_{V_{1}}(\xi)=\operatorname{tr}_{V_{2}}(\xi)$.

Proof. Observe that in $V_{l}$ we have $\operatorname{tr}_{v_{l}}(k)=k_{l}\left(1+q^{-1}\right)$ and $\operatorname{tr}_{v_{1}}(\xi)=\xi_{l}(1-q)$

Thus, each two-dimensional representation $V_{1}$ can be labelled by two numbers, $\left(k_{i}, \xi_{l}\right), k_{i}=\operatorname{tr}(k) /\left(1+q^{-1}\right)$ and $\xi_{l}=\operatorname{tr}(\xi) /(1-q)$, and denoted by $V_{\left(k_{i}, \xi_{l}\right)}$. The fusion rules of these two-dimensional representations are given by the following theorem.

THEOREM 3. $V_{\left(k_{1}, \xi_{1}\right)} \otimes V_{\left(k_{2}, \xi_{2}\right)}=V_{\left(k_{1}, k_{2}, \xi_{1} \xi_{2}\right)} \oplus V_{\left(q-1 k_{1} k_{2}, q \xi_{1} \xi_{2}\right)}$.

Proof. Denote the basis on the left-hand side by $v_{i}^{(1)} \otimes v_{j}^{(2)}(i, j=1,2)$. Then the basis for the right-hand side is

$$
\begin{aligned}
& \left\{v_{1}^{(1)} \otimes v_{1}^{(2)}, v_{1}^{(1)} \otimes v_{2}^{(2)}+k_{1} \xi_{2} y_{1} y_{2}^{-1} v_{2}^{(1)} \otimes v_{1}^{(2)}\right\}, \\
& \left\{v_{1}^{(1)} \otimes v_{2}^{(2)}-k_{2}^{-1} \xi_{1}^{-1} x_{1}^{-1} x_{2} v_{2}^{(1)} \otimes v_{1}^{(2)}, v_{2}^{(2)} \otimes v_{2}^{(2)}\right\}
\end{aligned}
$$

In this notation the fundamental representation given in Equation (4.4) is denoted by $V_{\left(q^{1,2}, q^{-3,2}\right)}$. The decomposition of its tensor product can be inferred from the following Corollary.

\section{COROLLARY.}

$$
V_{(k, \xi)}^{\otimes n}=\bigoplus_{l=0}^{n-1}\left(\begin{array}{c}
n-1 \\
l
\end{array}\right) V_{\left(k^{n} q-l,(-1) l \xi n^{\prime}{ }^{l}\right)} .
$$

We note that the multiplicities of the irreducible representations occurring in the decomposition $V_{(k, \xi)}^{\otimes n}$ form the Yang Hui-Pascal triangle. 
An interesting question is whether the two-dimensional representations other than the fundamental one would give rise to new braid group representations or not. The answer is no. This follows from Theorem 4.

THEOREM 4. The intertwining operator $R=\left(a_{i j}\right)$ between $V_{1} \otimes V_{2}$ and $V_{2} \otimes V_{1}$ (with $V_{1}, V_{2}$ any two two-dimensional representations) satisfying $R \circ \Delta(a)=$ $\Delta^{\prime}(A) \circ R$, or

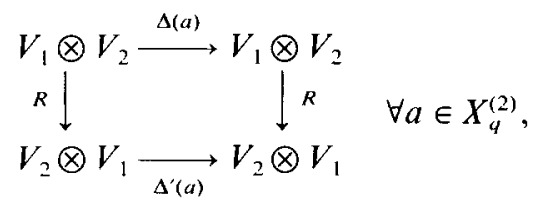

must be of the form

$$
R=\left(\begin{array}{cccc}
a_{11} & 0 & 0 & 0 \\
0 & a_{22} & a_{11}-B a_{22} & 0 \\
0 & a_{11}-A a_{22} & A B a_{22} & 0 \\
0 & 0 & 0 & a_{11}-(A+B) a_{22}
\end{array}\right)
$$

with

$$
A=\check{\zeta}_{1} k_{2} x_{1} / x_{2}, \quad B=k_{1}^{-1} \xi_{2}^{-1} y_{2} / y_{1} .
$$

Proof. Take $a=k, \xi, X$ and $Y$.

COROLLARY. The R-matrix satisfying the Yang-Baxter equations in End $\left(\otimes^{3} V_{\left(k_{i}, \xi_{1}\right)}\right)$ is given by Equation (4.7) with $a_{22} / a_{11}=\xi_{t} k_{t}=A=B^{-1}$ or the same $R$-matrix given in Equation (4.1) with $q$ replaced by $k_{1}^{-1} \xi_{i}^{-1}$.

To conclude, we note that if the last diagonal element of the $\check{R}$-matrix (1.1) or the $R$-matrix (4.1) is changed from $-q^{-1}$ to $q$, then we recover the standard Jones BGR and the associated quantum group $\mathrm{U}_{q}(\mathrm{sl}(2))$. For a generic $q$, the representations of the latter are in $1: 1$ correspondence to those of $\operatorname{sl}(2)$. In contrast, the representation theory of our $X_{4}(2)$ is quite distinct.

However, there are still some common features shared by the two cases. First, the characteristic polynomial of Jones' BGR and ours are, respectively,

$$
\begin{array}{ll}
(R-q)^{3}\left(R+q^{-1}\right)=0 & \text { (Jones), } \\
(R-q)^{2}\left(R+q^{-1}\right)^{2}=0 & \text { (ours). }
\end{array}
$$

The power of each factor in both cases corresponds to the fusion rule of the fundamental representation

$$
\begin{aligned}
& 2 \otimes 2=3 \oplus 1 \quad\left(\mathrm{sl}_{q}(2)\right), \\
& 2 \otimes 2=2^{\prime} \oplus 2^{\prime \prime} \quad\left(X_{q}(2)\right) .
\end{aligned}
$$

Here the numbers stand for the dimensionality of representations. Secondly, the $\breve{R}$-matrix for $X_{q}(2)$ can be written as

$$
\check{R}=q P_{1}-q^{-1} P_{2} \equiv \sum_{i=1}^{2} \rho_{l} P_{l},
$$


where $P_{1}$ and $P_{2}$ are projectors corresponding to the decomposition (4.10). (This follows from the fact that $[\check{R}, \Delta(a)]=0, \forall a \in X_{q}(2)$ and that $V_{0} \otimes V_{0}$ is completely reducible.) A similar expression is known to be true for $\mathrm{sl}_{4}(2)$ [2]. Because of this similarity, the same Yang-Baxterization rule suggested before [10] works for both cases. It is given by

$$
\check{R}(x)=x \check{R}^{+}-x^{-1}\left(\check{R}^{+}\right)^{-1},
$$

where $x$ is the spectral parameter.

\section{Appendix}

Let $\hat{X}_{4}(2)$ be the Hopf algebra obtained by removing the relations $X^{2}=Y^{2}=0$ from $X_{q}(2)$. In this Appendix, we study representations of $\hat{X}_{q}(2)$. We restrict ourselves to the so-called highest weight representations, i.e. there exists a $v_{0} \in V$ satisfying

$$
X v_{0}=0, \quad k v_{0}=k_{0} v_{0}, \quad \xi v_{0}=\xi_{0} v_{0} .
$$

Let $V$ be an irreducible finite-dimensional representation. Consider $v_{l}=Y^{l} v_{0}$ and suppose $\left\{v_{0}, v_{1}, \ldots, v_{m}\right\}$ is the maximal linearly independent set from $v_{l}$. Then $v_{m+1}=\sum_{l=0}^{m} c_{l} v_{l}$. Since $k \xi Y^{l}=(-1)^{l} Y^{l} k \xi$,

$$
\begin{aligned}
X v_{l} & =X Y^{l} v_{0}=\sum_{l=0}^{l-1} Y^{J}[X, Y] Y^{l-\jmath-1} v_{0} \\
& =\sum_{j=0}^{l-1}(-1)^{l-\jmath-1} Y^{l-1} \frac{k \xi-k^{-1} \xi^{-1}}{q-q^{-1}} v_{0} \\
& = \begin{cases}0, & l \equiv 0(\bmod 2), \\
\frac{k_{0} \xi_{0}-k_{0}^{-1} \xi_{0}^{-1}}{q-q^{-1}} v_{l-1}, & l \equiv 1(\bmod 2) .\end{cases}
\end{aligned}
$$

So, by its irreducibility, $V$ must be the space spanned by $\left\{v_{0}, v_{1}, \ldots, v_{m}\right\}$. Assume that $k_{0} \xi_{0} \neq k_{0}^{-1} \xi_{0}^{-1}$. (Otherwise, $X=0$ in this representation.) Applying $X$ to $v_{m+1}=\Sigma_{l=0}^{m} c_{l} v_{l}$, the resulting equation is consistent only if $m$ is odd. Namely, $V$ has to be even-dimensional. It is clear from Equation (A2) that $X^{2} v_{l}=0$ for any $l$. So $X^{2}=0$ in a nontrivial irreducible representation $V$. (By nontriviality of a representation, we mean the basis elements of $\hat{X}_{q}(2)$ act injectively.) Hence, we have proved the following theorem.

THEOREM. In any nontrivial irreducible representation $V$ of $\hat{X}_{q}(2)$ having both highest and lowest weight vectors, one always has $X^{2}=Y^{2}=0$ in $\operatorname{End}(V)$. Moreover, $\operatorname{dim} V=2$. 


\section{Acknowledgements}

We thank Profs. H. C. Lee, M. Jimbo, and L. A. Takhtajan for useful discussions. M. L. Ge and Y. S. Wu are particularly grateful to Prof. C. N. Yang for continual encouragement and enlightening discussions. M. L. Ge is supported in part by U.S. NSF grant \# PHY-89-08945 through ITP, Stony Brook. Y. S. Wu is supported in part by U.S. NSF grant \# PHY-8706501 and by the Monell Foundation through the Institute for Advanced Study. N. H. Jing acknowledges support by U.S. NSF grant \# DMS 8610730 through the Institute for Advanced Study.

\section{References}

1. Drınfeld, V. G., Dokl. Akad. Nauk. SSR 283, 1060 (1985); Proc. ICM-86, Berkeley, Academic Press, New York, pp. 798-820.

2. Jimbo, M., Lett. Math. Phys. 10, 63 (1985); 11, 247 (1986). A reference book, see Yang-Baxter Equation in Integrable System, edited by M. Jimbo, World Scientific, Singapore, 1990.

3. Yang, C. N., Phys. Rev. Lett. 19, 1312 (1967); Baxter, R. J., Exactly Solved Models in Statistical Mechanics, Academic Press, London, 1982.

4. Moore, G. and Seiberg, N., Nuclear Phys. B313, 16 (1989); Comm. Math. Phys. 123, 177 (1989); Alvarez-Gaume, L., Gomez, G., and Sierra, G., Phys. Lett. 220B, 142 (1989); CERN TH5267/88.

5. Witten, E., Comm. Math. Phys. 121, 351 (1989).

6 Witten, E., IAS, HEP-89/77.

7 Jimbo M., Comm. Math. Phys. 102, 537 (1986).

8. Reshetikhın, N. Yu., LOMI preprint E-4-87, 1987.

9. Ge, M. L.. Wang. L. Y., Xue. K., and Wu. Y. S. Internat. J. Modern Phys. 4, 3351 (1989); Ge, $M$ L. and Xue, K., ITP-SB-90-20.

10. Ge. M. L., Wu. Y. S, and Xue, K., ITP-SB-90-02.

11. Lee, H C., Couture, M., and Schmeing, N. C., Chalk River preprint.

12. Faddeev, L. D., Reshetikhin, N. Yu., and Takhtajan, L., in C. N. Yang and M. L. Ge (eds), Braid Group. Knot Theory and Statstical Mechanics; Takhtajan, L., Introduction to Quantum Group, Nankaı Mathematical Physics Lectures, to be published by World Scientific, Singapore.

13. Lee, H. C., Proc. NATO ARW on Phys. and Geometry, Lake Takoe, July 1989.

14 Kauffman, L. H., Knots, abstract tensors and the Yang-Baxter equation, Preprint, 1989. 